\title{
Acute myeloid leukemia of donor origin after allogeneic stem cell transplantation from a sibling who harbors germline XPD and XRCC3 homozygous polymorphisms
}

Hilda Rachel Diamond ${ }^{1 *}$, Maria Helena Ornellas ${ }^{2}$, Alberto Orfao ${ }^{3}$, Bernadete E Gomes ${ }^{1}$, Mércia M Campos ${ }^{1}$, Teresa S Fernandez ${ }^{4}$, Roberto I da Silva ${ }^{2,5,6}$, Gilda Alves ${ }^{5}$, Claudia Lage ${ }^{6}$, Dayse A da Silva², Arthur Moellmann-Coelho ${ }^{7}$, Geydson S da Cruz ${ }^{7}$, Luis Fernando Bouzas ${ }^{8}$ and Eliana Abdelhay ${ }^{9}$

\begin{abstract}
A 54-year-old woman was diagnosed with infiltrative ductal breast carcinoma. Two years after treatment, the patient developed an acute myeloid leukemia (AML) which harbored del(11q23) in 8\% of the blast cells. The patient was submitted for allogeneic stem cell transplantation (aSCT) from her HLA-compatible sister. Ten months after transplantation, she relapsed with an AML with basophilic maturation characterized by $C D 45^{\text {low }} \mathrm{CD} 33^{\text {high }}$, $\mathrm{CD}_{117^{+}}, \mathrm{CD} 13^{-1+}, \mathrm{HLA}$ Dr ${ }^{\text {high }}, \mathrm{CD} 123^{\text {high }}$, and CD203c $\mathrm{C}^{+}$blast cells lacking expression of CD7, CD10, CD34, CD15, CD14, CD56, CD36, CD64, and cytoplasmic tryptase. Karyotype analysis showed the emergence of a new clone with $\mathrm{t}(2 ; 14)$ and FISH analysis indicated the presence of $M L L$ gene rearrangement consistent with del(11q23).

Interestingly, AML blast cell DNA tested with microsatellite markers showed the same pattern as the donor's, suggesting that this AML emerged from donor cells. Additionally, polymorphisms of the XPA, XPD, XRCC1, XRCC3 and RAD51 DNA repair genes revealed three unfavorable alleles with low DNA repair capacity.

In summary, we report the first case of AML involving XPD and XRCC3 polymorphisms from donor origin following allogeneic stem cell transplantation and highlight the potential need for careful analysis of DNA repair gene polymorphisms in selecting candidate donors prior to allogeneic stem cell transplantation.
\end{abstract}

Keywords: immunophenotype, cytogenetics, DNA repair, donor origin leukemia

\section{Background}

Breast cancer is the most frequent malignancy in women [1]. Over recent decades overall survival of breast cancer patients has increased considerably as a result of earlier diagnosis and increasing use of adjuvant therapies [2,3]. Nevertheless, the risk of developing a secondary cancer increases as a long-term complication related to the use of cytotoxic DNA-targeted antiproliferative drugs and hormone therapy with or without radiotherapy $[4,5]$.

Among other complications, a small proportion of all breast cancer survivors subsequently develop acute

\footnotetext{
* Correspondence: hrdiamond@hotmail.com

'Laboratory of Immunology, Bone Marrow Transplantation Unit, National Cancer Institute, Praça Cruz Vermelha $n^{\circ} 23,6^{\circ}$ andar. Centro, Rio de Janeiro, RJ, 20230-130, Brazil

Full list of author information is available at the end of the article
}

myeloblastic leukemia (AML), preceded or not by a preleukemic myelodysplastic syndrome (MDS) [5]. Secondary AML has many morphological and cytogenetic variants because transforming mutations leading to the disease are heterogeneous and occur in an early multipotential hematopoietic cell that retains the potential to differentiate into virtually every hematopoietic lineage [6].

Here we report a rare case of a donor-related secondary AML with basophilic maturation post-allogeneic stem cell transplantation in a patient with prior history of secondary AML derived from primary breast cancer chemotherapy. To our knowledge this is the first case reported in the literature of a donor cell-derived AML secondary to breast cancer treatment and allogeneic stem cell transplantation associated with unfavourable DNA repair gene polymorphisms. 


\section{Case presentation}

A 54-year-old woman was submitted for mastectomy in May 2004 because of an infiltrative ductal breast carcinoma, with negative nodal infiltration and without expression of hormone receptors. After surgery, she was treated with cyclophosphamide $\left(600 \mathrm{mg} / \mathrm{m}^{2}\right)$ and doxorubicin $(60$ $\mathrm{mg} / \mathrm{m}^{2} ; 4$ cycles) followed by adjuvant radiotherapy. In December 2006, the patient presented with fever, anemia, and gingival bleeding. Peripheral blood data revealed anemia (hemoglobin level of $7.7 \mathrm{~g} / \mathrm{L}$ ) and thrombocytopenia $\left(12 \times 10^{9}\right.$ platelets/L $)$ with an increased white blood cell count $\left(35.4 \times 10^{9}\right.$ leucocytes $\left./ \mathrm{L}\right)$, with $70 \%$ blasts. A bone marrow aspirate sample showed diffuse infiltration by blast cells and diagnosis of AML M5 according to the French-American-British (FAB) classification was made. On immunophenotypic criteria, blast cells were positive for CD33, CD117, CD13, and HLA-DR.

Cytogenetic studies performed on a bone marrow aspirate sample using standard culture methods and GTG banding revealed a normal 46, XX[25] karyotype at the time of the diagnosis of secondary AML prior to allogeneic stem cell transplantation (allo-SCT). Cytogenetic markers for secondary AML [del(11)(q23), del(5q)/-5 or del(7q)/-7] were further investigated by interphase fluorescence in situ hybridization (iFISH) using the LSI MLL (11q23) dual color, LSI D7S486 spectrum orange/CEP7 spectrum green, LSI EGR1 spectrum orange/LSID5S23:D5S721 spectrum green, and LSI CSF 1R spectrum orange/LSID5S23:D5S721 spectrum green (Vysis, Abbott Laboratories, USA) iFISH probes and showed $8 \%$ cells carrying del(11q23) in the absence of abnormalities of both chromosomes 5 and 7 . The patient was treated with $\mathrm{AraC}$ and idarrubicin [7-9] and complete remission was attained. Consolidation was performed with cytarabin-arabinose (high dose Ara-C) plus filgrastin, as prophylaxis for leucopenia.

After two cycles of consolidation, she was submitted to an allo-SCT from her HLA-compatible sister. The conditioning regimen for the allo-SCT consisted of bussulfan and cyclosphosphamide. At day +48 , she developed acute graft versus host disease (aGVHD), and was treated with corticosteroids and cyclosporine (CSA). At day +280 , an inguinal $4 \times 3 \mathrm{~cm}$ mass appeared and the presence of malignant cells was revealed upon biopsy. At day +300 , a bone marrow aspirate showed $50 \%$ blasts. Cellular and molecular analyses were performed in parallel on this sample. Immunophenotyping of bone marrow cells confirmed the presence of $\mathrm{CD} 45^{\text {low }}$ $\mathrm{CD} 33^{\text {high }}, \mathrm{CD} 117^{+}, \mathrm{CD} 13^{-/+}$, HLA Dr ${ }^{\text {high }} \mathrm{CD} 123^{\text {high }}$ blast cells, lacking expression of CD7, CD10, CD34, CD15, CD14, CD56, CD36, and CD64 (Figure 1A-F),
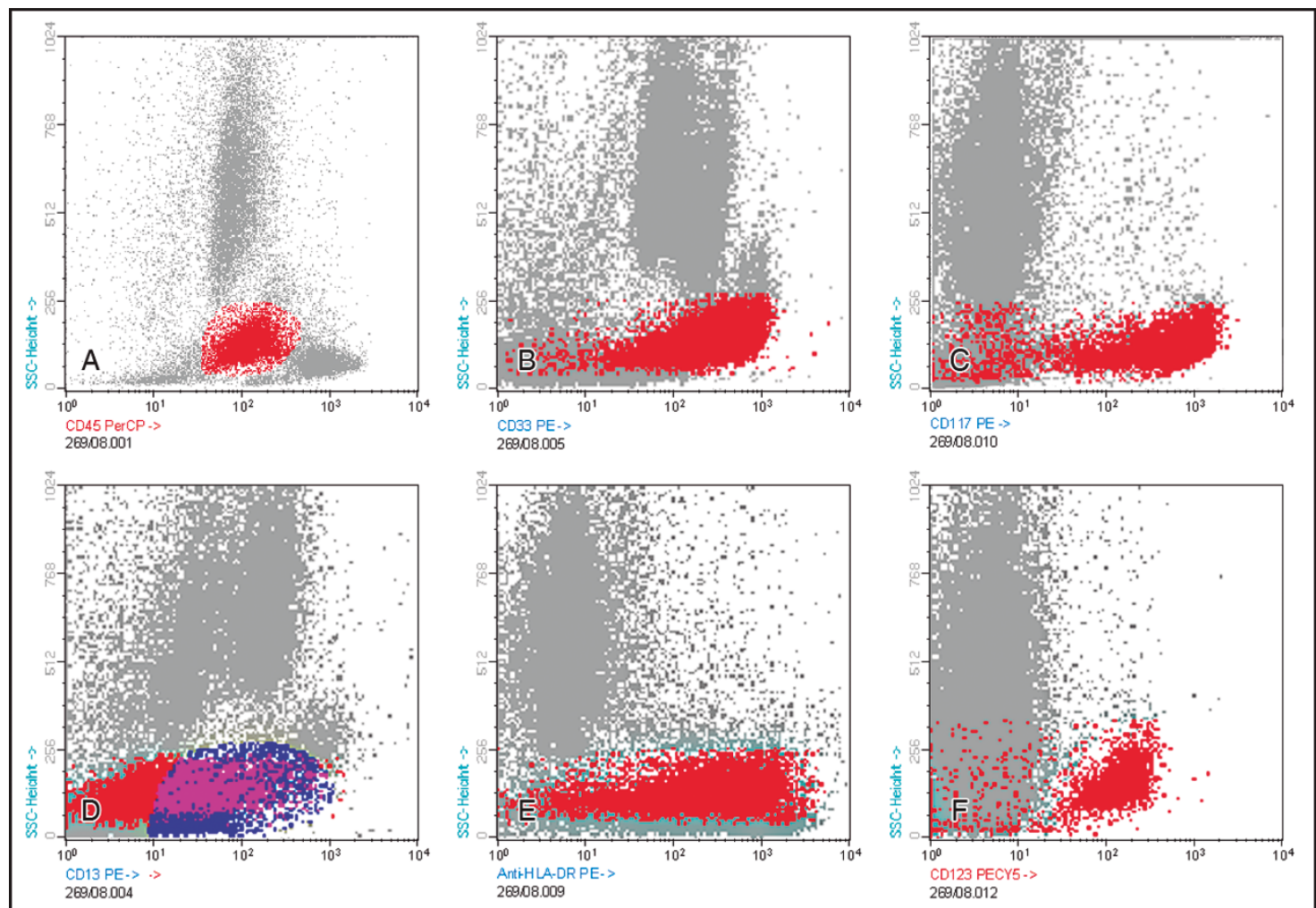

Figure 1 Bivariate flow cytometry dot plots showing the immunoreactivity pattern of blast cells (gated on CD45 vs. SSC) for CD45 ${ }^{\text {low }}=$ $25 \%$ (panel A), CD33 = 24\% (panel B), CD117 = 24\% (panel C), CD13 = 16\% (panel D), HLA-DR = 25\% (panel E), and CD123= 25\%(panel F). 
suggesting maturation into the basophil $v s$. mast cell lineages. Further immunocytochemical stainings were performed showing CD203c expression in the absence of cytoplasmic tryptase (Figure 2A and 2B); these together with the high expression for CD123 were consistent with basophilic maturation.

Microsatellite and PCR-RFLP analyses were performed on genomic DNA from mononuclear cells of the patient pre-transplant, of the AML blast cell sample obtained after transplantation, and of the donor bone marrow cells. The 11 tested microsatellite markers are often used in forensic medicine for individual identification. As displayed in Table 1, 7 of the analyzed loci (D21S11, D7S820, CSF1PO, D3S1358, Vwa, D13S317, and TPOX) were informative and showed the coincidence of profile between AML blasts after transplantation and the donor's cells, supporting full engraftment of the stem cell transplant as well as the donor cell-origin of the AML blasts.

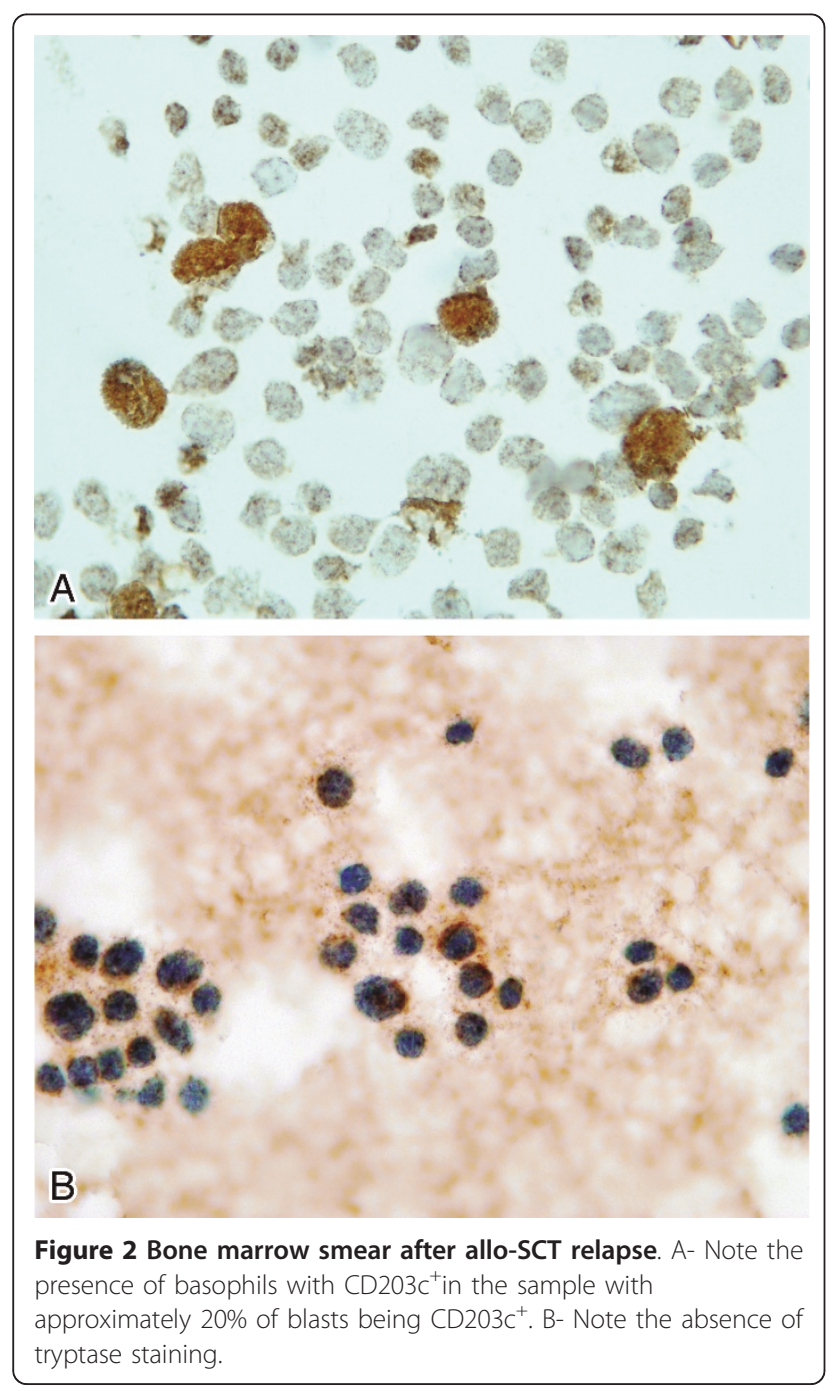

Genetic polymorphisms of five relevant human DNA repair genes (XPA, XPD, XRCC1, XRCC3, and RAD51) were further analyzed by PCR-RFLP [10-15] on the secondary AML blast DNA and compared to a pre-transplant DNA sample from the patient and to DNA from the donor. Analysis of leukemia-prone polymorphic alleles (XPA A23G, XPD Lys751Gln, XRCC1 Arg399Gln, $X R C C 3$ Thr241Met, and RAD51 G135C) revealed an $X P D$ and $X R C C 3$-deficient heterozygous pre-SCT patient with normal alleles for XPA, XRCC1, and RAD51 DNA repair functions (Table 2 and Figure 3). Donor polymorphisms were different, harboring both $X P D$ - and XRCC3-deficient homozygosis. Once microsatellite analysis indicated post-transplant total chimerism, post-SCT patient cells were shown to have acquired the genotypic markers of the donor's poorer DNA repair functions (Table 2 and Figure 3).

The patient was then treated with idarubicin, cytosine arabinoside (ARA-C) with no response. At that time the immunophenotypic study showed the same profile, but conventional cytogenetics revealed the emergence of a new clone: 46, XX, t(2;14)(q37;q22)[2]/46, XX [33](Figure 4). Treatment was modified and FLAG [fludarabin, ARA-C, and granulocyte colony-stimulating factor (GCSF)] was started. After 18 days the patient had hematopoietic recovery with $3 \%$ blasts. Twenty-one days after FLAG, a donor lymphocyte infusion (DLI) was given, which was followed by GVHD, and the patient was treated with corticosteroids. The patient was submitted to a second DLI four months later. At day +14 after this second DLI, the karyotype post-DLI was normal, 46, XX [43], but FISH analysis indicated the presence of $M L L$ gene rearrangements consistent with del(11q23) in 5\% of the cells. The myelogram showed $62 \%$ blasts. Rescue therapy with high dose topotecan and ARA-C was started and administered for five days without haematologic response; peripheral blood infiltration by blast cells rose to $80 \%$ after 14 days. Palliative support began and the patient died after 17 months of stem cell infusion.

\section{Discussions and Conclusions}

Here we report a case of a secondary AML developing from donor-derived cells in a breast cancer patient who underwent allogeneic stem cell transplantation. Donor cell leukemia is a rare although well-recognized disease entity following SCT that occurs as the result of oncogenic transformation of apparently normal donor hematopoietic cells in the transplant recipient.

Many studies have reported an increased risk of breast cancer patients to develop leukemia after chemotherapy, radiotherapy and G-CSF administration $[1,5,16,17]$. Because of this, risk estimates on the eventual development of post-treatment AML/MDS have to be cast when deciding a patient's treatment. In these studies, 
Table 1 Microsatellite markers in the patient's pre and post-transplant haematopoietic cells compared with the donor's

\begin{tabular}{llll}
\hline Marker & Patient pre-SCT & Patient AML & Donor \\
\hline D21S11 & 28 & $\mathbf{2 8}$ & $\mathbf{2 8}$ \\
& 31.2 & $\mathbf{2 9}$ & $\mathbf{2 9}$ \\
\hline D75820 & 8 & $\mathbf{8}$ & $\mathbf{8}$ \\
& 10 & $\mathbf{9}$ & $\mathbf{9}$ \\
\hline CSF1PO & 11 & $\mathbf{8}$ & $\mathbf{8}$ \\
& 12 & $\mathbf{1 1}$ & $\mathbf{1 1}$ \\
\hline D351358 & 15 & $\mathbf{1 5}$ & $\mathbf{1 5}$ \\
& 15 & $\mathbf{1 7}$ & $\mathbf{1 7}$ \\
\hline TH01 & 8 & 8 & 8 \\
& 9 & 9 & 9 \\
\hline D13S317 & 9 & $\mathbf{1 1}$ & $\mathbf{1 1}$ \\
& 13 & $\mathbf{1 3}$ & $\mathbf{1 3}$ \\
\hline D165539 & 13 & 13 & 13 \\
& 14 & 14 & 14 \\
\hline Vwa & 16 & $\mathbf{1 7}$ & $\mathbf{1 7}$ \\
& 18 & $\mathbf{1 8}$ & $\mathbf{1 8}$ \\
\hline TPOX & 8 & $\mathbf{9}$ & $\mathbf{9}$ \\
& 11 & $\mathbf{1 2}$ & $\mathbf{1 2}$ \\
\hline D5S818 & 12 & 12 & 12 \\
\hline FGA & 13 & 13 & 23 \\
\hline 21 & 23 & 21 & 13 \\
\hline
\end{tabular}

Informative and coincident markers are identified in bold.

increased risk of AML/MDS has been reported for cases treated with alkylating agents and anthracyclines. Posttreatment secondary leukemias associated with prior administration of alkylating agents (e.g., cyclophosphamide) typically differ from those arising after treatment with DNA topoisomerase II inhibitors, such as anthracyclines. Accordingly, AML's developing after topoisomerase II inhibitors are given typically show an early onset, and display monocytic and myelomonocytic features in association with abnormalities of chromosomes 11 and
21 (especially balanced translocations involving the $11 \mathrm{q} 23$ and 21q22 regions). Whereas those arising after treatment with alkylating agents frequently show neutrophil/granulocytic maturation together with abnormalities of chromosome 5 and 7. These changes are seen in the absence of chromosomal translocations and the leukemias emerge much later after therapy [18].

Exposure to radiotherapy may further increase the risk for AML [19-21]. In turn, Smith et al. [20] found the M4/M5 subtypes to be more frequent in patients receiving intense treatment regimens and concluded that this could be the result of cyclophosphamide-induced promotion of a doxorubicin-associated leukemogenic effect. In the case reported here, the association of chemotherapy and radiotherapy protocols most probably played a role in the development of AML. Secondary AML outcomes are thus believed to arise from genomic instability (i.e., deletions, mutations, translocations) induced by therapy-associated DNA damage [22,23]. Two different hypotheses remain which could contribute to explain the development of secondary AML: a truly stochastic event, or individual differences on cancer susceptibility [22]. The latter appears to better explain the case reported here. In this regard, previous reports have described polymorphisms conferring sensitivity to chemotherapy, which may contribute to the incidence of secondary AML outcomes [24-26]. Alternatively, it is possible that germline variations in DNA repair genes may also enhance the risk of therapy-induced secondary AML in patients carrying DNA-repair deficient genes [27]. In fact it has also been shown that single nucleotide polymorphisms (SNP) in DNA repair genes may code malfunctioning proteins, in association with an increased predisposition to cancer and the response of leukemia patients following chemotherapy $[12,28,29]$. Here we investigated five alleles of DNA repair genes already ascribed to susceptibility to leukemia (XRCC1, $X P A, X P D, X R C C 3$ and $R A D 51)$. As expected, polymorphic alleles found by genotyping in both the patient

Table 2 DNA repair polymorphisms in the patient's pre- and post-transplantation (AML) cells and donor's cells

\begin{tabular}{|c|c|c|c|c|}
\hline Polymorphism & $\begin{array}{l}\text { DNA repair } \\
\text { mechanism }\end{array}$ & $\begin{array}{l}\text { Patient cells (pre- } \\
\text { transplantation) }\end{array}$ & $\begin{array}{l}\text { AML cells (post- } \\
\text { transplantation) }\end{array}$ & Donor cells \\
\hline XPA A23G & NER & Homozygous for optimal DRC & Homozygous for optimal DRC & Homozygous for optimal DRC \\
\hline XPD Lys751Gln & NER & Heterozygous deficient DRC & Homozygous for deficient DRC & $\begin{array}{l}\text { Homozygous for deficient } \\
\text { DRC }\end{array}$ \\
\hline XRCC1 Ar399GIn & BER & Homozygous for optimal DRC & Homozygous for optimal DRC & Homozygous for optimal DRC \\
\hline $\begin{array}{l}\text { XRCC3 } \\
\text { Thr243Met }\end{array}$ & HRR & Heterozygous for deficient DRC & Homozygous for deficient DRC & $\begin{array}{l}\text { Homozygous for deficient } \\
\text { DRC }\end{array}$ \\
\hline RAD51 G135C & HRR & Homozygous for optimal DRC & Homozygous for optimal DRC & Homozygous for optimal DRC \\
\hline
\end{tabular}

DRC - DNA repair capacity

NER - Nucleotide Excision Repair

BER - Base Excision Repair

HRR - Homologous Recombination Repair 


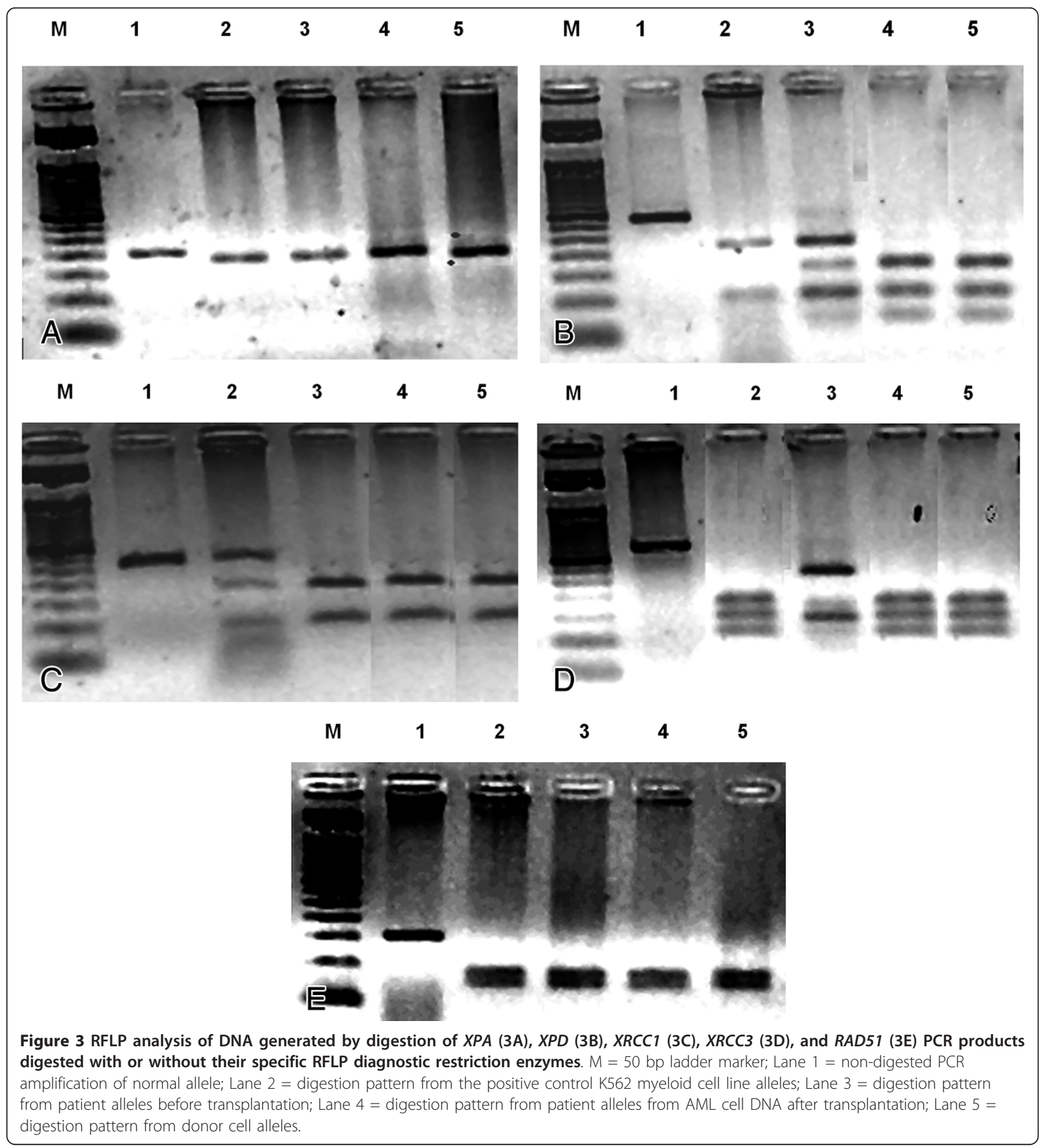

post-SCT hematopoietic cells and the donor's cells were the same, revealing successful transplant, which could also explain the otherwise observed resistance to treatment and clinical evolution. In turn, pre-SCT patient cells were heterozygous for allelic variants in the $X P D$ and $X R C C 3$ genes that are associated with lower repair capacity, conspicuously bearing on susceptibility to breast cancer and chemotherapy-related leukemia
(AML) in this patient. Consistent with our observations, Allan et al. [12] showed that individuals carrying at least one $X P D$ - Lys751Gln allele were more likely to have an adverse prognosis following chemotherapy. The XPD Gln751 polymorphic protein was shown to fail in engaging apoptosis in chemotherapy-damaged cells, thus avoiding elimination of mutated myeloid precursors. In order to search for an association of the poor outcome 


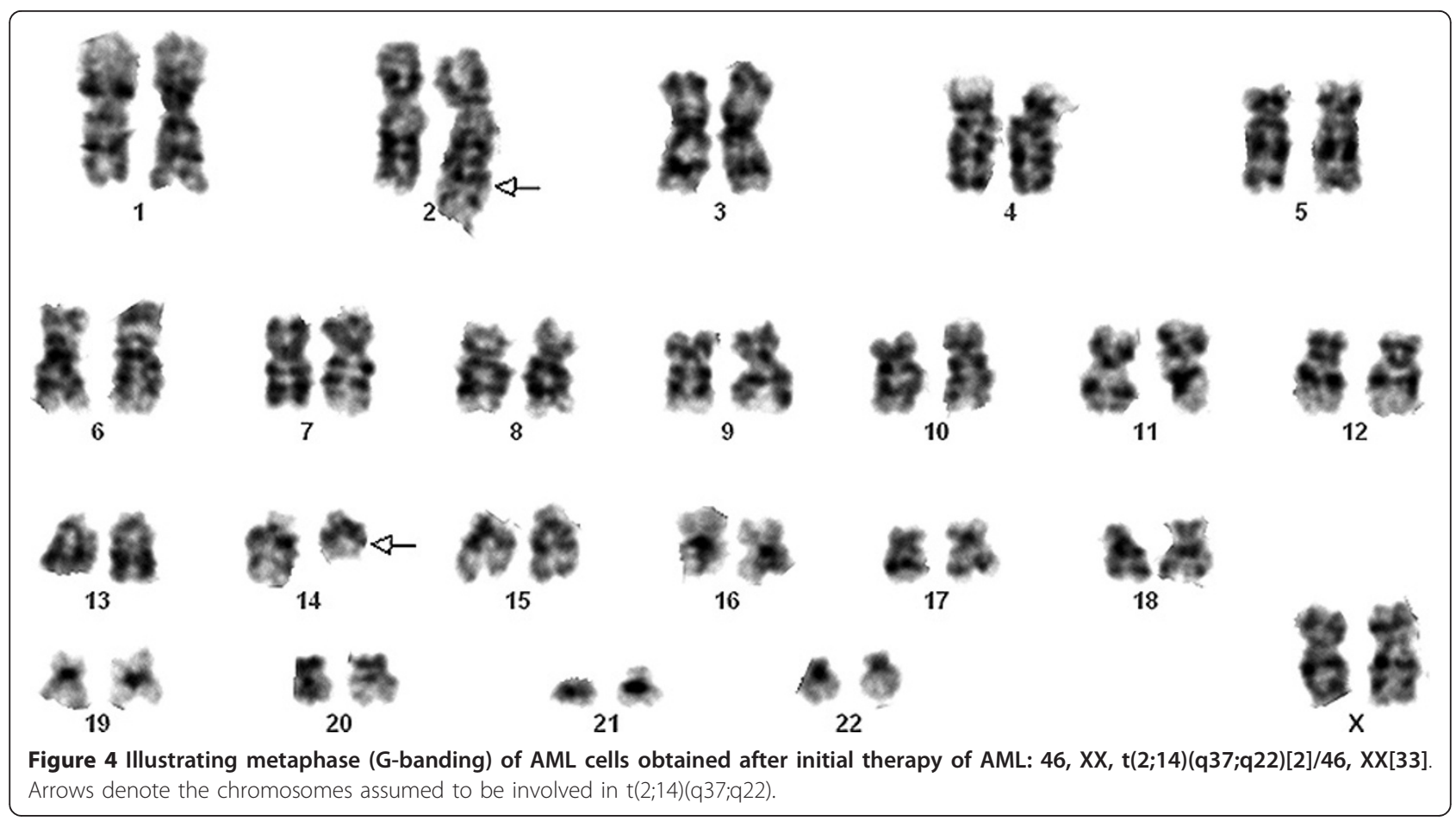

of the patient's secondary post-SCT leukemia, the same gene set of polymorphisms was screened for in the donor's DNA. Remarkably, donor DNA genotyped homozygous not only for the XPD (Lys751Gln) suboptimal allele, but also for the XRCC3-deficient allele (Thr243Met). Both post-SCT blast cells and donor DNA were coincident in XPD- and XRCC3-deficient homozygosity, supporting a donor origin for the leukemic blasts.

The $X R C C 3$ protein plays a critical role in Homologous Recombination Repair (HRR) accounting for repair of DNA double-strand breaks (DSB). Whenever it is deficient, unsealed or misrepaired breaks can generate oncogenic chromosomal translocations. When found together, allelic variants in Lys751Gln $X P D$ and Thr241Met XRCC3 polymorphisms have been associated with both a worse repair capacity and resistance to apoptosis $[11,30]$. Once introduced into the patient's drug-intoxicated bone marrow, disrupted DNA repair and apoptosis pathways in the donor's cells may have accumulated unrepaired damages while escaping apoptosis, thus boosting aggressiveness. Indeed, this is further supported by the observation of a switch from normal to highly abnormal post-SCT of the patient's karyotype and FISH analysis. This supports the theory that in donor origin leukemia, the host environment in which the original malignancy developed could trigger an oncogenic process in donor cells, favored by the immunosuppressive status after transplantation, especially because the donor is still healthy [31,32].
An additional interesting finding is the maturation observed phenotypically towards the basophilic versus mast cell lineages based on coexpression of CD203c and both CD123, CD117 [33]. Cases of de novo AML with predominant basophilic and/or mastocytic, cell phenotypes are uncommon and they account for only $4-5 \%$ of all cases of acute nonlymphocytic leukemia [34,35]. Although acute basophilic leukemia (ABL) has been long diagnosed as such, current knowledge of this specific AML subtype remains limited [36].

In conclusion, here we describe a very rare case of donor origin AML from a sibling who harbored germline $X P D$ and XRCC3 homozygous polymorphisms in a breast cancer patient following chemotherapy. The blasts showed SNP profiles of the donor including susceptible alleles for unfavorable genotypes in DNA repair genes. Determination of the donor's DNA repair genotype, capable of stimulating genetic instability in a diseased recipient, could be important for future transplantation procedures and therefore should be investigated further. DNA repair mechanisms are responsible for maintenance of the genome integrity avoiding additional mutations in key cell cycle regulation genes which are responsable for "leukemization". To ensure that DNA repair mechanisms are properly working, more donor cell gene polymorphisms or mutations should be studied. After the interpretation of results, a reference panel of low DNA repair capacity polymorphisms or mutations should be included in the international guidelines for screening donor DNA before SCT. 


\section{Consent}

The Ethics Committee of the National Cancer Institute performed in accordance with the ethical standards laid down in the 1964 Declaration of Helsinki, approved this study (\# of registration 41/11). The written informed consent was obtained from the sister's patient.

A copy of the written consent is available for review by the Editor-in-Chief of this journal.

\section{Acknowledgements}

Ministério da Saúde/INCA, FAPERJ.

\section{Author details}

${ }^{1}$ Laboratory of Immunology, Bone Marrow Transplantation Unit, National Cancer Institute, Praça Cruz Vermelha $n^{\circ} 23,6^{\circ}$ andar. Centro, Rio de Janeiro, RJ, 20230-130, Brazil. ²Department of Pathology, Rio de Janeiro State University, Avenida Manoel de Abreu 444, $4^{\circ}$ andar -Patologia Geral, Rio de Janeiro, RJ, 20550-170, Brazil. ${ }^{3}$ Cancer Research Centre (IBMCC-CSIC/USAL), University of Salamanca, Centro de Investigación del Cáncer Paseo de la Universidad de Coimbra s/n37007 Salamanca, Spain. ${ }^{4}$ Laboratory of Cytogenetics, Bone Marrow Transplantation Unit, National Cancer Institute, Praça Cruz Vermelha $n^{\circ} 23,6^{\circ}$ andar. Centro, Rio de Janeiro, RJ, 20230-130, Brazil. ${ }^{5}$ Laboratory of Applied Genetics, Hematology Service, National Cancer Institute, Praça Cruz Vermelha $n^{\circ} 23,6^{\circ}$ andar. Centro, Rio de Janeiro, RJ, 20230-130, Brazil. ${ }^{6}$ Program of Molecular and Structural Biology, Carlos Chagas Filho Biophysics Institute, Rio de Janeiro Federal University, CCS BLOCO G - SALA G0-031, ILHA DA CIDADE UNIVERSITÁRIA, Rio de Janeiro, RJ, 21941-902, Brazil. ${ }^{7}$ Hematology Service, National Cancer Institute, Praça Cruz Vermelha $n^{\circ} 23,8^{\circ}$ andar. Centro, Rio de Janeiro, RJ, 20230-130, Brazil. ${ }^{8}$ Clinical Division, Bone Marrow Transplantation Unit, National Cancer Institute, Praça Cruz Vermelha $n^{\circ} 23,7^{\circ}$ andar. Centro, Rio de Janeiro, RJ, 20230-130, Brazil. 'Stem Cell Laboratory, Bone Marrow Transplantation Unit, National Cancer Institute, Praça Cruz Vermelha $n^{\circ} 23,6^{\circ}$ andar. Centro, Rio de Janeiro, RJ, 20230-130, Brazil.

\section{Authors' contributions}

$H R D$, designed the paper and wrote the paper. AO, performed immunocytochemical stainings and reviewed the manuscript. BEG and MMC, performed flow cytometric immunophenotyping. TSF performed cytogenetic and FISH analysis. RIS, GA, CL and DAS, performed the molecular biology studies. AM-C and GSC, were responsible of the patient's treatment and conceived the study. GSC and MHO, carried out acquisition of data's patient. $\mathrm{CL}, \mathrm{GA}$ and $\mathrm{MHO}$ were responsible for manuscript review. EA and LFB carried out their critical interpretations. All authors read and approved the final manuscript.

\section{Competing interests}

The authors declare that they have no competing interests.

Received: 24 August 2011 Accepted: 27 September 2011 Published: 27 September 2011

\section{References}

1. Le Deley MC, Suzan F, Cutuli B, Delaloge S, Shamsaldin A, Linassier C Clisant S, de Vathaire F, Fenaux P, Hill C: Anthracyclines, mitoxantrone, radiotherapy, and granulocyte colony-stimulating factor: risk factors for leukemia and myelodysplastic syndrome after breast cancer. J Clin Oncol 2007, 25:292-300.

2. Budman DR, Berry DA, Cirrincione $C T$, Henderson IC, Wood WC, Weiss RB, Ferree CR, Muss HB, Green MR, Norton L, Frei E: Dose and dose intensity as determinants of outcome in the adjuvant treatment of breast cancer. The Cancer and Leukemia Group B. J Natl Cancer Inst 1998, 90:1205-1211.

3. Polychemotherapy for early breast cancer: an overview of the randomised trials. Early Breast Cancer Trialists' Collaborative Group. Lancet 1998, 352:930-942.

4. Srivastava A, Murari M, Datta NR: Early occurrence of acute myeloid leukemia following adjuvant radiotherapy and higher cumulative dose of cyclophosphamide in carcinoma breast. Indian J Cancer 2004, 41:178-180.

5. Praga C, Bergh J, Bliss J, Bonneterre J, Cesana B, Coombes RC, Fargeot $P$, Folin A, Fumoleau $P$, Giuliani R, Kerbrat $P$, Hery M, Nilsson J, Onida F, Piccart M, Shepherd L, Therasse P, Wils J, Rogers D: Risk of acute myeloid leukemia and myelodysplastic syndrome in trials of adjuvant epirubicin for early breast cancer: correlation with doses of epirubicin and cyclophosphamide. J Clin Oncol 2005, 23:4179-4191.

6. Lichtman MA: The stem cell in the pathogenesis and treatment of myelogenous leukemia: a perspective. Leukemia 2001, 15:1489-1494.

7. Vogler WR, Velez-Garcia E, Weiner RS, Flaum MA, Bartolucci AA, Omura GA, Gerber MC, Banks PL: A phase III trial comparing idarubicin and daunorubicin in combination with cytarabine in acute myelogenous leukemia: a Southeastern Cancer Study Group Study. J Clin Oncol 1992, 10:1103-1111.

8. Cassileth PA, Harrington DP, Appelbaum FR, Lazarus HM, Rowe JM, Paietta E, Willman C, Hurd DD, Bennett JM, Blume KG, Head DR, Wiernik PH: Chemotherapy compared with autologous or allogeneic bone marrow transplantation in the management of acute myeloid leukemia in first remission. N Engl J Med 1998, 339:1649-1656.

9. INCA/MS CONDUTAS DO INCA/MS. Leucemia Mielóide Aguda em Adultos. Rev Bras Cancerol 2002, 48:313-315.

10. Levy-Lahad E, Lahad A, Eisenberg S, Dagan E, Paperna T, Kasinetz L, Catane R, Kaufman B, Beller U, Renbaum P, Gershoni-Baruch R: A single nucleotide polymorphism in the RAD51 gene modifies cancer risk in BRCA2 but not BRCA1 carriers. Proc Natl Acad Sci USA 2001, 98:3232-3236.

11. Wu X, Zhao H, Wei Q, Amos Cl, Zhang K, Guo Z, Qiao Y, Hong WK, Spitz MR: XPA polymorphism associated with reduced lung cancer risk and a modulating effect on nucleotide excision repair capacity. Carcinogenesis 2003, 24:505-509.

12. Allan JM, Smith AG, Wheatley K, Hills RK, Travis LB, Hill DA, Swirsky DM, Morgan GJ, Wild CP: Genetic variation in XPD predicts treatment outcome and risk of acute myeloid leukemia following chemotherapy. Blood 2004, 104:3872-3877.

13. Seedhouse C, Faulkner R, Ashraf N, Das-Gupta E, Russell N: Polymorphisms in genes involved in homologous recombination repair interact to increase the risk of developing acute myeloid leukemia. Clin Cancer Res 2004, 10:2675-2680.

14. Dufloth RM, Costa S, Schmitt F, Zeferino LC: DNA repair gene polymorphisms and susceptibility to familial breast cancer in a group of patients from Campinas, Brazil. Genet Mol Res 2005, 4:771-782.

15. Nouspikel T: DNA repair in mammalian cells: Nucleotide excision repair: variations on versatility. Cell Mol Life Sci 2009, 66:994-1009.

16. Leone G, Pagano L, Ben-Yehuda D, Voso MT: Therapy-related leukemia and myelodysplasia: susceptibility and incidence. Haematologica 2007, 92 (10):1389-1398

17. Hershman D, Neugut Al, Jacobson JS, Wang J, Tsai WY, McBride R, Bennett CL, Grann VR: Acute Myeloid Leukemia or Myelodysplastic Syndrome Following Use of Granulocyte Colony-Stimulating Factors During Breast Cancer Adjuvant Chemotherapy. J Natl Cancer Inst 2007 99:196-205.

18. Pedersen-Bjergaard J, Philip P: Two different classes of therapy-related and de-novo acute myeloid leukemia? Cancer Genet Cytogenet 1991, 55:119-124.

19. Park MJ, Park YH, Ahn HJ, Choi W, Paik KH, Kim JM, Chang YH, Ryoo BY, Yang SH: Secondary hematological malignancies after breast cancer chemotherapy. Leuk Lymphoma 2005, 46:1183-1188.

20. Smith RE, Bryant J, DeCillis A, Anderson S: Acute myeloid leukemia and myelodysplastic syndrome after doxorubicin- cyclophosphamide adjuvant therapy for operable breast cancer: The national surgical adjuvant breast and bowel project experience. J Clin Oncol 2003, 21:1195-1204.

21. Crump M, Tu D, Shepherd L, Levine M, Bramwell V, Pritchard K: Risk of acute leukemia following epirubicin-based adjuvant chemotherapy: a report from the National Cancer Institute of Canada Clinical Trials Group. J Clin Oncol 2003, 21:3066-3071.

22. Larson RA: Etiology and management of therapy-related myeloid leukemia. Hematology Am Soc Hematol Educ Program 2007, 453-459.

23. Rowley JD, Olney HJ: International workshop on the relationship of prior therapy to balanced chromosome aberrations in therapy-related 
myelodysplastic syndromes and acute leukemia: overview report. Genes Chromosomes Cancer 2002, 33:331-345.

24. Larson RA, Wang Y, Banerjee M, Wiemels J, Hartford C, Le Beau MM, Smith MT: Prevalence of the inactivating 609C- $T$ polymorphism in the $\mathrm{NAD}(\mathrm{P}) \mathrm{H}$ :quinone oxidoreductase (NQO1) gene in patients with primary and therapy-related myeloid leukemia. Blood 1999, 94:803-807.

25. Allan JM, Wild CP, Rollinson S, Willett EV, Moorman AV, Dovey GJ, Roddam PL, Roman E, Cartwright RA, Morgan GJ: Polymorphisms in glutathione S-transferase P1 is associated with susceptibility to chemotherapy-induced leukemia. Proc Natl Acad Sci USA 2001, 98:11592-11597.

26. Naoe T, Takeyama K, Yokozawa T, Kiyoi H, Seto M, Uike N, Ino T, Utsunomiya A, Maruta A, Jin-nai I, Kamada N, Kubota Y, Nakamura H, Shimazaki C, Horiike S, Kodera Y, Saito H, Ueda R, Wiemels J, Ohno R: Analysis of genetic polymorphism in NQ01, GST-M1, GST-T1, and CYP3A4 in 469 Japanese patients with therapy-related leukemia/ myelodysplastic syndrome and de novo acute myeloid leukemia. Clin Cancer Res 2000, 6:4091-4095.

27. Seedhouse C, Bainton R, Lewis M, Harding A, Russell N, Das-Gupta E: The genotype distribution of the XRCC1 gene indicates a role for base excision repair in the development of therapy-related acute myeloblastic leukemia. Blood 2002, 100:3761-3766.

28. Goode EL, Ulrich CM, Potter JD: Polymorphisms in DNA repair genes and associations with cancer risk. Cancer Epidemiol Biomarkers Prev 2002, 11:1513-1530, Erratum in: Cancer Epidemiol. Biomarkers Prev 2003; 12 (10):1119.

29. Kuptsova N, Kopecky KJ, Godwin J, Anderson J, Hoque A, Willman CL, Slovak ML, Ambrosone CB: Polymorphisms in DNA repair genes and therapeutic outcomes of AML patients from SWGOG clinical trials. Blood 2007, 109:3936-3944

30. Gu J, Zhao H, Dinney CP, Zhu Y, Leibovici D, Bermejo CE, Grossman HB, Wu X: Nucleotide excision repair gene polymorphisms and recurrence after treatment for superficial bladder cancer. Clin Cancer Res 2005, 11:1408-1415.

31. Flynn CM, Kaufman DS: Donor cell leukemia: insight into cancer stem cells and the stem cell niche. Blood 2007, 109:2688-2692.

32. Xiao H, Shi J, Luo Y, Tan Y, He J, Xie W, Zhang L, Wang Y, Liu L, Wu K, Yu X, Cai $Z$, Lin $M$, Ye $X$, Huang H: First report of multiple CEBPA mutations contributing to donor origin of leukemia relapse after allogeneic hematopoietic stem cell transplantation. Blood 2011, 117:5257-5260.

33. Horny HP, Sillaber C, Menke D, Kaiserling E, Wehrmann M, Stehberger B, Chott A, Lechner K, Lennert K, Valent P: Diagnostic value of immunostaining for tryptase in patients with mastocytosis. Am J Surg Pathol 1998, 22:1132-1140.

34. Quattrin N: Follow up of sixty-two cases of acute basophilic leukemia. Biomedicine 1978, 28:72-79.

35. Peterson LC, Parkin JL, Arthur DC, Brunning RD: Acute basophilic leukemia: a clinical, morphologic and cytogenetic study of eight cases. Am J Clin Pathol 1991, 96:160-170.

36. Seth T, Vora A, Bhutani M, Ganessan K, Jain P, Kochupillai V: Acute basophilic leukemia with $\mathrm{t}(8 ; 21)$. Leuk Lymphoma 2004, 45:605-608, Erratum in: Leuk Lymphoma 2004; 45(6):1311.

\section{doi:10.1186/1756-8722-4-39}

Cite this article as: Diamond et al: Acute myeloid leukemia of donor origin after allogeneic stem cell transplantation from a sibling who harbors germline XPD and XRCC3 homozygous polymorphisms. Journal of Hematology \& Oncology 2011 4:39.

\section{Submit your next manuscript to BioMed Central and take full advantage of:}

- Convenient online submission

- Thorough peer review

- No space constraints or color figure charges

- Immediate publication on acceptance

- Inclusion in PubMed, CAS, Scopus and Google Scholar

- Research which is freely available for redistribution 